\title{
Synopsis of Most Relevant Articles on Cardiac Arrhythmias
}

\author{
Section Editor: Bruno Papelbaum¹ \\ Papelbaum B iD https://orcid.org/0000-0001-7154-7001 \\ 1. Hospital da Beneficência Portuguesa - São Paulo (SP) - Brazil. \\ E-mail: brpapel@gmail.com
}

\section{A randomized trial of subcutaneous versus transvenous defibrillator therapy: The PRAETORIAN trial. D-LBCT01-02. Late-Breaking Clinical Trials. Randomized Trials*}

Complications related to transvenous leads remain a concern among patients treated with a transvenous implantable cardioverter-defibrillator (TV-ICD). The subcutaneous ICD (S-ICD) was designed to reduce leadrelated complications by utilizing an entirely extrathoracic placement of the system, circumventing heart and vasculature. No randomized, controlled trials that compare both therapies have been published to date.

The trial, which was conducted by Reinoud Knops, Academisch Medisch Centrum, Universiteit van Amsterdam, (Amsterdam, Netherlands) and colleagues, was the first to compare the safety and efficacy of S-ICDs and TV-ICDs.

A total of 849 patients with a class I or IIa indication for ICD therapy and without the need for pacing from 39 centers in the USA and Europe, were randomized and followed until a median of four years. Out of the total number of patients, 426 patients were assigned to receive an S-ICD and 423 were assigned to a TV-ICD, with similar baseline characteristics in both groups. The trial population was $20 \%$ female, with a median age of 63 years (55-70 years), and 69\% had ischemic cardiomyopathy with a median left ventricular ejection fraction of $30 \%(25-35 \%)$.

Results of the PRAETORIAN trial showed that, in a general ICD-population, the subcutaneous ICD was noninferior to the transvenous ICD with regards to major ICD-related adverse events with significantly less leadrelated complications in patients treated with the S-ICD. The primary end point of the trial was a combination of device-related complications and inappropriate shocks during a four-year median follow-up, and an upper 95\% confidence limit of 1.45 for the hazard ratio of the primary end point was used to test for noninferiority. Secondary end points included death from any cause (including sudden cardiac death) and appropriate ICD therapy. Since the rate of most lead-related complications of TV-ICDs occur after more than four years, patient cohort of the PRAETORIAN trial will be followed for a further four years.

According to the study team, the results highlight that S-ICDs are an important treatment option for patients in need of an implantable cardioverter defibrillator (ICD) without pacing indication. 


\section{Persistent atrial fibrillation ablation with a contact force sensing catheter: the prospective multicenter PRECEPT trial*}

The safety and effectiveness of catheter ablation for paroxysmal AF has been extensively studied. In contrast, robust evidence is lacking on persistent AF (PsAF) ablation. PRECEPT is the first FDA-regulated study to evaluate the safety and effectiveness of radiofrequency catheter ablation (RFA) for PsAF using a porous tip contact force (CF)-sensing catheter.

The study was a prospective, multicenter, nonrandomized trial, taking place at 27 sites in the USA and Canada, involving patients who had documented symptomatic persistent $\mathrm{AF}$ and nonresponse or intolerance to at least one antiarrhythmic drug (AAD) (class I or III). The trial primary safety endpoint was the incidence of primary adverse events occurring within seven days of the initial and repeat ablation procedures; the primary effectiveness endpoint was freedom from documented recurrence of AF, atrial flutter (AFL) or atrial tachycardia (AT) episodes of 30 seconds or longer, and freedom from additional five-failure modes at 15 months, which included: acute procedural failure, use of nonstudy catheter, repeat procedures, use of new/higher AAD dose or surgical ablation.

Ablation included pulmonary vein isolation with additional left-atrial targets, permitted at the investigators' discretion. Arrhythmia recurrences were assessed by electrocardiogram, Holter, and monthly transtelephonic monitoring for up to 15 months postablation, with a three-month medication adjustment period followed by a three-month therapy consolidation period. Primary effectiveness success was defined as freedom from documented AF/atrial tachycardia (AT)/atrial flutter (AFL) recurrence and additional five-failure modes at 15 months. Clinical success was defined as freedom from documented symptomatic AF/AT/AFL recurrence. Patients' quality-of-life (QoL) improvement was evaluated using the Atrial Fibrillation Effect on Quality-of-Life (AFEQT) score.

A total of 381 patients were enrolled in the study between July 27 2016, and February 6 2018, of which 348 had the investigational catheter inserted and comprised the safety population. All participants in the safety population underwent RF ablation. Four participants had missing three months data for safety assessment and thus were removed from the primary safety endpoint analysis. The effectiveness population comprised 333 participants after exclusion of 14 participants who did not meet inclusion criteria and one participant who was ablated with a nonstudy catheter.

According to the results presented, the Kaplan-Meier estimate of primary effectiveness at 15 months stood at 61.7\% (95\% CI: 56.2-67.2\%) of patients reporting freedom from recurrences of AF, AT or AFL, acute procedural failure, use of nonstudy catheter, repeat procedures, use of new/higher AAD dose or surgical ablation. This meant that the study performance goal $40.0 \%$ for primary effectiveness was met. Clinical success was reported in $80.4 \%$ of patients.

A total of 14 primary adverse events for 13 participants were reported on the safety outcomes. The primary adverse event rate was $3.8 \%$ and one-sided exact $97.5 \%$ upper confidence bound was $6.4 \%$, significantly less than the specified performance goal of $16.0 \%$.

The authors conclude that "the PRECEPT study demonstrated the clinical safety and effectiveness of PsAF ablation using CF-sensing technologies with protocol-defined effectiveness of $62 \%$ and clinical success of $80 \%$. The PAE [primary adverse event] rate was within the acceptable and expected range and similar to that for paroxysmal AF ablation. Comparison with other multicenter studies suggests individualized ablation approach base on patient's clinical presentation may optimize treatment outcome".

* Mansour M, Calkins H, Osorio J, Pollak SJ, Melby D, Marchlinski FE, et al. Persistent atrial fibrillation ablation with contact force sensing catheter: The prospective multicenter PRECEPT Trial. JACC Clin Electrophysiol. Forthcoming 2020. 


\section{Leftbundle branch pacingas a novel strategy forcardiacresynchronization therapy: results from international LBBP collaborative study group: D-LBCT02-01. Late-Breaking Clinical Trials Innovation Boulevard*}

Cardiac resynchronization therapy (CRT) using biventricular pacing (BVP) or His bundle pacing (HBP) is effective in patients with heart failure (HF), bundle branch block or RV pacing. Intraseptal left bundle branch pacing (LBBP) has been reported as an alternative option for CRT. The aim of the study was to assess the feasibility and outcomes of LBBP in CRT candidates in an international, multicenter, collaborative study.

Left bundle branch pacing was attempted in patients with LVEF $<50 \%$ and indications for CRT/pacing at eight international centers. Indications, procedural and pacing parameters, NYHA class, HF hospitalization, echocardiographic data, and lead complications were assessed. The procedure was performed as a rescue or primary approach to achieve CRT utilizing SelectSecure 3830 pacing lead. Left bundle branch capture was assessed by the presence of: LBB potentials, paced RBBB morphology, nonselective (NS) to selective (S) or myocardial capture, peak LV activation time (pLVAT) $<90 \mathrm{~ms}$ in V6, response to programmed stimulation.

Left bundle branch pacing was attempted in 325 pts and CRT was successfully achieved in 277 (85\%); failure due to incomplete CRT 27, failed deep septal deployment 21. Age $71 \pm 13$ years, female 37\%, HTN 68\%, DM 38\%, CAD 47\%. Ischemic CMP 36\%, nonischemic CMP 59\%; LVEF < 35\% in 65\% pts. QRS morphology at baseline: LBBB 42\%, RBBB 18\%, IVCD 12\%, RVP 13\%, narrow 16\%. Procedure and fluoroscopy duration were $105 \pm 54$ and $19 \pm 16 \mathrm{~min}$. Left bundle branch pacing threshold and $\mathrm{R}$-wave amplitudes were $0.76 \pm 0.5 \mathrm{~V}$ at $0.5 \mathrm{~ms}$ and $11.5 \pm 6.8 \mathrm{mV}$ at implant and remained stable during mean $\mathrm{f} / \mathrm{u}$ of $6 \pm 5$ months. Left bundle branch pacing resulted in significant narrowing of QRS from $156 \pm 32 \mathrm{~ms}$ to $137 \pm 22 \mathrm{~ms}$ ( $\mathrm{p}<0.0001)$ with pLVAT of $83 \pm 16$ ms. Ejection fraction (EF) improved from $32 \pm 9$ to $44 \pm 11 \%(\mathrm{p}<0.0001)$. Left bundle branch capture evidence, echo and clinical outcomes and complications will be presented.

The authors concluded that LBBP is feasible, safe and provides an alternative option for cardiac resynchronization therapy. It also provides remarkably low and stable pacing thresholds. Insights from this study may provide better electrophysiologic understanding of LBBP and its role in CRT. Randomized, controlled trials comparing conduction system pacing to BVP will be necessary.

*Callans DJ, Tedrow UB. D-LBCT02 Late-Breaking clinical trials innovation boulevard. Heart Rhythm. Forthcoming 2020. https://doi. org/10.1016/j.hrthm.2020.04.042

\section{PULSED AF: first human experience and acute procedural outcomes using a novel pulsed field ablation system: D-LBCT02-03. Late-Breaking Clinical Trials Innovation Boulevard*}

Pulmonary vein isolation (PVI) is a cornerstone of catheter ablation for atrial fibrillation (AF), and rapidly evolving technologies are poised to improve the safety and efficacy of this procedure. Irreversible electroporation using pulsed field ablation may be highly selective for myocardial tissue, improving the efficiency of PVI while potentially minimizing collateral damage to nonmyocardial tissues.

PULSED AF trial is a nonrandomized, prospective, multicenter, global, pre-market clinical study performed in Australia, Canada, United States and Europe. The study evaluates the Medtronic PulseSelect system, a PFA system that delivers bipolar, biphasic pulsed electric fields through a circular multielectrode array catheter to perform PVI.

The patients included were undergoing first-time ablation for either paroxysmal or persistent AF (less than one year). The study endpoints include AF recurrence $>30 \mathrm{~s}$, procedural safety within 30 days postablation and 
effectiveness such as acute PVI ablation with the system. Atrial fibrillation monitoring is being performed by weekly transtelephonic transmission and intermittent Holter at six and 12 months. Patients will ultimately be followed for 12-month postablation.

Baseline characteristics in 14 out of 20 patients (due to covid-19) included male gender (50\%), paroxysmal AF in $93 \%$, left atrial diameter $36.7 \pm 6.7 \mathrm{~mm}$ and a CHA2DS2VASc of $2.4 \pm 2.1$. Results of the PULSED-AF study suggest that PFA delivers ablation as effective as RF ablation, while providing a safer and more efficient procedure. Results show that acute electrical isolation was achieved in $100 \%$ of patients and there were no tamponades, strokes or phrenic nerve injuries. Upon conclusion, the study will report the rate of arrhythmia-free survival at 12 months, and prespecified secondary and ancillary endpoints, including procedural outcomes, quality of life and arrhythmic symptoms.

The authors of the study point to a future presentation of full 12-month patient follow-ups results and conclude that PFA can achieve acute isolation in $100 \%$ of treated veins without any serious adverse events.

*Callans DJ, Tedrow UB. D-LBCT02 Late-Breaking clinical trials innovation boulevard. Heart Rhythm. Forthcoming 2020. https://doi. org/10.1016/j.hrthm.2020.04.042

\section{Point-by-point pulsed field ablation (+/- radiofrequency ablation) to treat atrial fibrillation: a first-in-human trial. D-LBCT02-04. Late-Breaking Clinical Trials Innovation Boulevard ${ }^{\star}$}

The tissue selectivity of pulsed field ablation (PFA) provides safety advantages over radiofrequency (RF) ablation. One-shot PFA catheters for pulmonary vein isolation (PVI) exist, but they don't permit flexible lesion sets - e.g., linear lesions. In a first-in-human trial, a novel lattice-tip ablation catheter able to deliver either focal $\mathrm{RF}$ or PF energy was used to treat paroxysmal atrial fibrillation (PAF) or persistent atrial fibrillation (PerAF) with either: i) PFA posteriorly and RFA anteriorly (RF/PF), or ii) PFA only (PF/PF).

The 8Fr lattice catheter has a compressible $9 \mathrm{~mm}$ nitinol tip and is used with a custom mapping system and RF \& PF generators (Sphere-9, Prism-1, HexaGen \& HexaPulse, respectively; Affera Inc). Toggling between energy sources, point-by-point PV encirclement was performed using biphasic PFA (2-5 s; 24-32 A) posteriorly, and either temp-controlled irrigated RFA (Tmax $73{ }^{\circ} \mathrm{C} ; 5$ s) or PFA anteriorly. Pulmonary vein isolation was confirmed with bidirectional pacing, and adenosine or after a 20 min wait. Linear lesions were with PFA or RFA.

It was a nonrandomized trial with patients enrolled at three centers (11 operators), a 76-pt cohort (age $59 \pm 9.9$ years; $\mathrm{M} / \mathrm{F}=50 / 26 ; \mathrm{PAF} / \mathrm{PerAF}=55 / 21)$ underwent either RF/PF (40 pts; $47.4 \pm 16.2$ lesions/pt) or PF/PF (36 pts; $53.1 \pm 14.4$ lesions/pt) ablation. The PVI therapy duration time (transpiring from first to last lesion) was $22.6 \pm 8.3 \mathrm{~min} / \mathrm{pt}$. Linear lesions included $13 \mathrm{mitral}(4 \mathrm{RF} / 1 \mathrm{RF}+\mathrm{PF} / 8 \mathrm{PF}), 33 \mathrm{LA}$ roof $(12 \mathrm{RF} / 21 \mathrm{PF})$ and $43 \mathrm{CTI}(35 \mathrm{RF} / 8 \mathrm{PF})$ lines, with therapy duration times of $5.1 \pm 3.6,1.8 \pm 2.4$ and $2.4 \pm 2.2 \mathrm{~min} / \mathrm{pt}$, respectively. All lesion sets were acutely successful. Total fluoroscopy time was $4.7 \pm 3.5 \mathrm{~min}$. There were no device complications; there were four vascular injuries. Postprocedure esophagogastroduodenoscopy (EGD) revealed minor mucosal thermal injury in 2 of $36 \mathrm{RF} / \mathrm{PF}$ and 0 of $24 \mathrm{PF} / \mathrm{PF}$ pts. Brain MRI revealed DWI+/FLAIR- and DWI+/FLAIR+ asymptomatic lesions in 4 and 3 of 52 pts, respectively; the initial ACT was lower in MRI-positive (255 $\pm 26 \mathrm{~s})$ vs MRI-negative $(349 \pm 84$ s) pts (p<0.00001). Only 1 pt (of 29; 3.4\%) with ACT > 300 had an MRI-positive lesion (DWI+/FLAIR-).

The authors concluded that the focal lattice catheter could safely and rapidly ablate AF using either a combined $\mathrm{RF} / \mathrm{PF}$ approach (capitalizing on the safety of PFA and the years of experience with RFA) or a PF-only approach. Questions addressed were how durable lesions are and what are the clinical outcomes. 


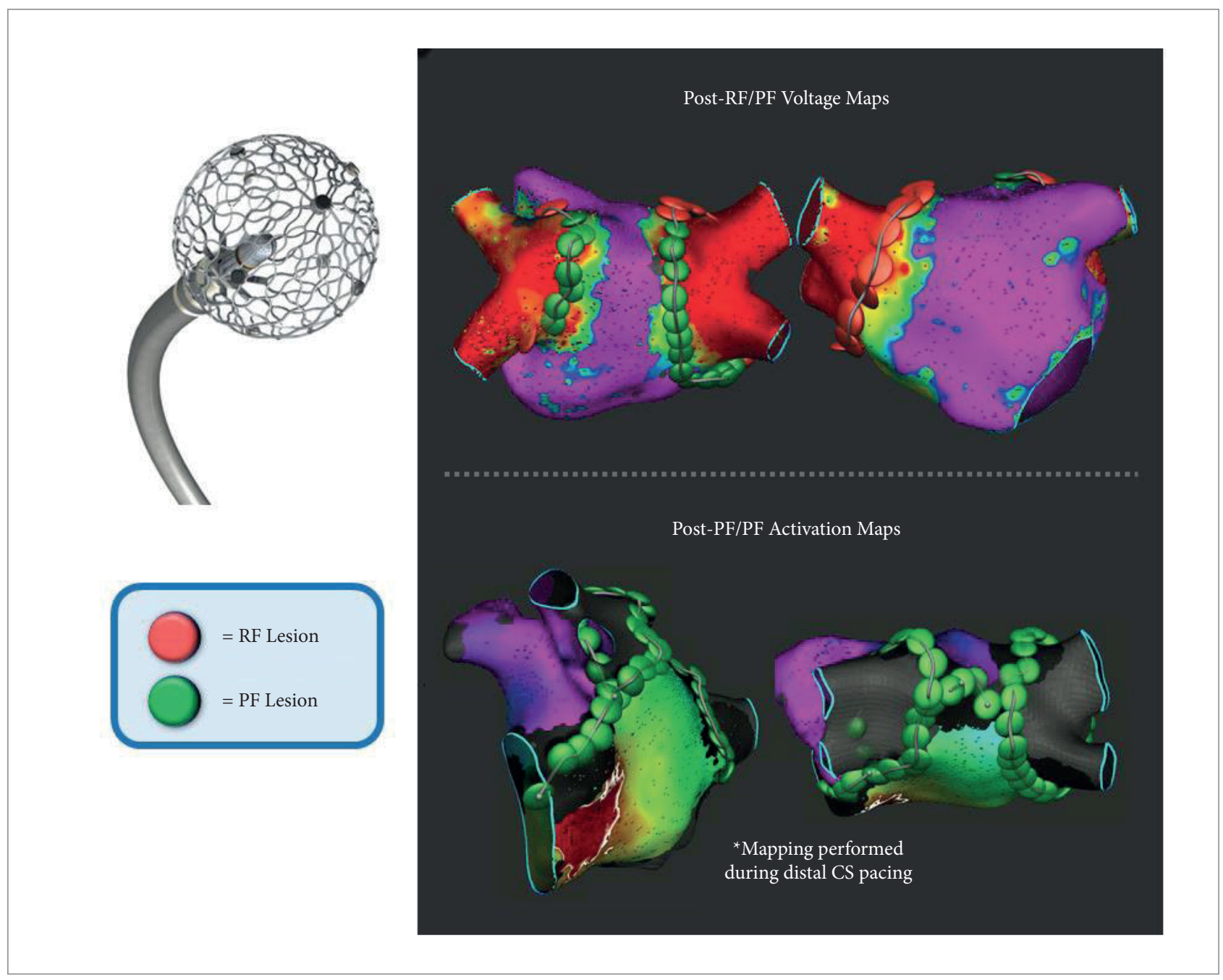

*Reddy VY, Anter E, Rackauskas G, Peichl P, KoruthJS, Petru J, et al. A lattice-tip focal ablation catheter that toggles between radiofrequency and pulsed field energy to treat atrial fibrillation: a first-in-human trial. Circ Arrhythm Electrophysiol. 2020. https://doi.org/10.1161/ CIRCEP.120.008718

\section{Predicted benefit of the implantable cardioverter defibrillator: the MADIT-ICD Benefit Score. D-LBCT04-02. Late-breaking clinical trials this could change my practice*}

The benefit of the ICD for primary prevention is not uniform. Authors present the first prediction model for ICD-benefit that integrates the predicted risk for arrhythmic events vs. nonarrhythmic mortality in all ICD patients enrolled in the MADIT trials.

For the purpose of this study they established the MEGA-MADIT database, combining all landmark MADIT trials between 1997 and 2011 (MADIT-II, MADIT-Risk, MADIT-CRT, and MADIT-RIT), with 4531 patients. Best-subsets Fine and Gray analysis was used to develop prognostic models for ventricular tachyarrhythmic events (VTE) (event of interest), VT $\geq 170 \mathrm{bpm}$, or death without prior VTE (competing event). Both competing risk models were subsequently integrated to form the MADIT-ICD Benefit Score.

Eight clinical factors (male, age $<75$ years, prior nonsustained VT, LVEF $<20 \%$, no aspirin, beta-blocker, and digitalis or diuretic use) were identified as predictors of VTE; and five clinical factors (age $\geq 75$ years, prior CABG, diabetes, HF hospitalization, amiodarone use) as predictors of death without prior VTE. Both competing models were 
combined to form the MADIT-ICD Benefit Score. Increasing ICD Benefit Score was directly correlated with the four-year predicted risk of VTE, and inversely correlated with the four-year predicted risk of death without a prior arrhythmia. Thus, patients in the highest MADIT-ICD benefit group had the highest predicted risk of VTE and the lowest predicted risk of death without a prior arrhythmia (29 vs. 7\%, respectively; $p<0.0001)$, with the highest potential for life-years saved by the ICD. In contrast, patients in the lowest MADIT-ICD benefit group had a similar risk of VTE vs. death without a prior arrhythmia (19 vs. $18 \%$, respectively; $\mathrm{p}=0.72$ ), with the lowest potential for life-years saved by the ICD. Internal validation confirmed model stability with similar C indices (0.66 and 0.67).

Based on the landmark MADIT trials, the authors propose the MADIT-ICD Benefit Score which predicts the likelihood of ICD benefit through personalized assessment of the risk of VTE weighed against the risk of nonarrhythmic mortality. The score may be used for shared decision-making in potential ICD candidates.

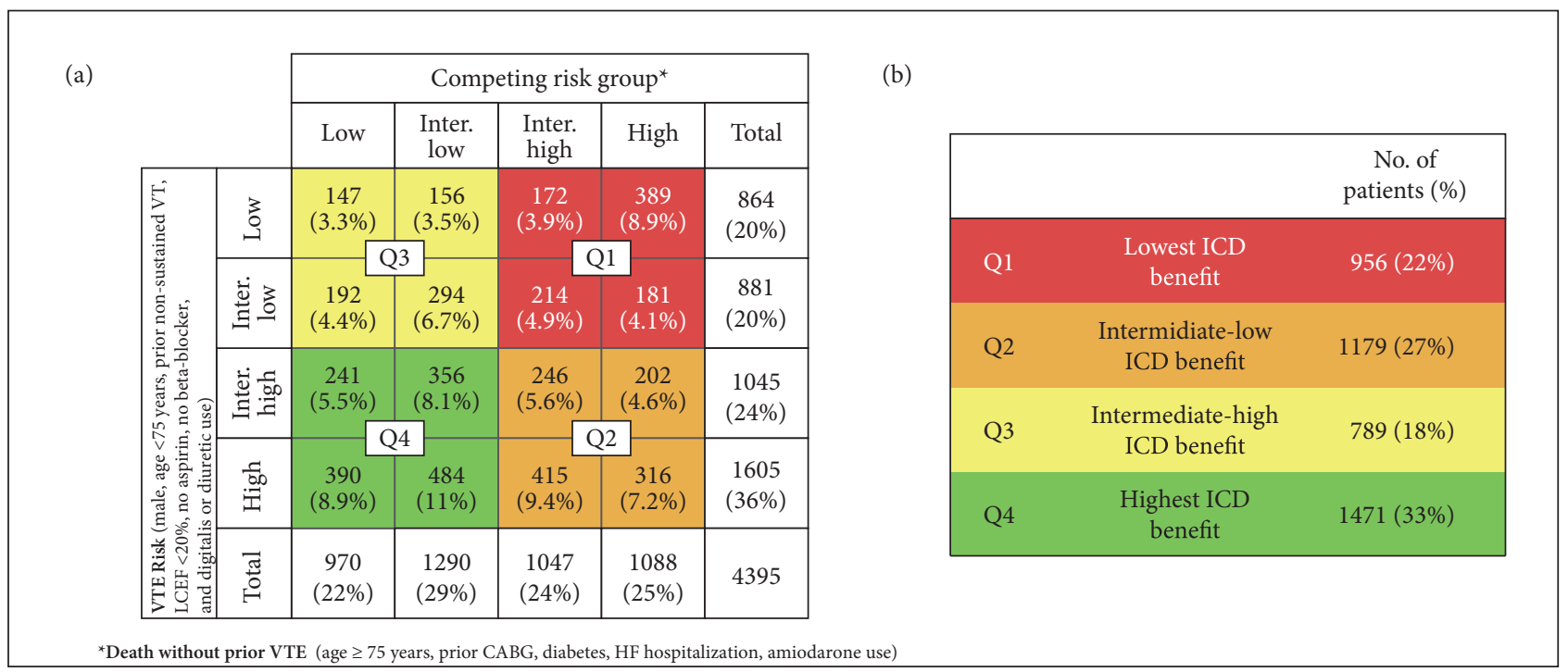

*Marrouche NF, Tung R. D-LBCT04 Late-Breaking clinical trials this could change my practice. Heart Rhythm. Forthcoming 2020. https:// doi.org/10.1016/j.hrthm.2020.04.044 